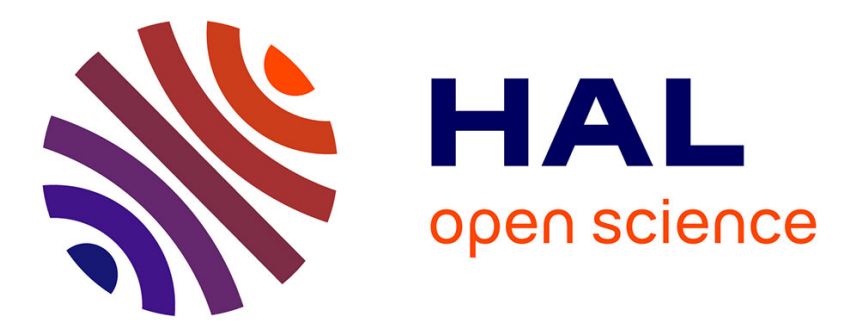

\title{
Pouvait-on habiter un camp de concentration sous le nazisme?
}

André-Frédéric Hoyaux

\section{To cite this version:}

André-Frédéric Hoyaux. Pouvait-on habiter un camp de concentration sous le nazisme?. Travaux de l'Institut de Géographie de Reims, 2006, 29-30 (115-118), pp.123-136. 10.3406/tigr.2003.1467 . halshs-00331404

\section{HAL Id: halshs-00331404 https://shs.hal.science/halshs-00331404}

Submitted on 24 Jun 2013

HAL is a multi-disciplinary open access archive for the deposit and dissemination of scientific research documents, whether they are published or not. The documents may come from teaching and research institutions in France or abroad, or from public or private research centers.
L'archive ouverte pluridisciplinaire HAL, est destinée au dépôt et à la diffusion de documents scientifiques de niveau recherche, publiés ou non, émanant des établissements d'enseignement et de recherche français ou étrangers, des laboratoires publics ou privés. 


\title{
TRAVAUX DE L'INSTITUT DE GEOGRAPHIE DE REIMS
}

\author{
Hoyaux André-Frédéric
}

Université Bordeaux 3 - UMR 6588 MITI, équipe TIDE.

\author{
Pouvait-on habiter un camp de concentration sous le nazisme $!^{1}$
}

«Il y a de l'être là où il y a résistance »

M. Dorra, 2001, 23.

En ces périodes de commémoration de la libération des camps d'Auschwitz, à l'heure où les médias compilent des interviews d'anciens déportés et nous présentent des documentaires sur la réalité des camps, il est intéressant, au-delà des contingences habituelles liées aux existences de tout un chacun, de comprendre comment ces rescapés ont fait pour résister à la violence physique et symbolique du pouvoir nazi, là où d'autres sont morts submergés par l'acharnement totalitaire. De comprendre comment ces femmes et ces hommes ont réussis à Ettre au-delà même du non-sens totalitaire, c'est-à-dire à Habiter au-delà de la violence qui régit la destruction volontaire de la relation au monde.

Une telle analyse part du principe que pour appréhender l'habitation de l'homme en période de paix, on peut utilement poser sa question à travers sa négation, c'est-à-dire à travers l'impossibilité réservée à des êtres humains d'exister en tant qu'ils sont des êtres qui construisent leur monde spatial, social et temporel et arrivent à toute fin utile à donner du sens à ce monde pour eux-mêmes et pour ceux qui les entourent. Ainsi, les déportés qui ont su résister aux principes mortifères des camps sont ceux qui ont su garder une relation avec un monde réel ou imaginé hors de ces camps. Ils ont su préserver ce hors là nécessaire à toute existence, c'est-à-dire créer une singularité territoriale au sein d'un non-lieu.

En effet, si habiter, c'est se construire territorialement dans le monde et pouvoir ainsi s'approprier, s'identifier, se projeter à chaque instant sur un lieu du Monde ; alors les rescapés sont ceux qui ont su éclairer par leur intensité pratique (par le faire du corps) et pragmatique (par le dire et le penser de l'esprit) une entité spatiale (un endroit, un paysage), sociale (une personne, les amis du travail) ou temporelle (un souvenir, un à-venir), que celle-ci soit proche ou lointaine, et à laquelle ils ont voué une préoccupation, une empathie ou un intérêt particulier.

Et si habiter, c'est aussi se constituer ontologiquement, c'est-à-dire se donner du sens par ce que l'on est ou croît être en ce monde et donner du sens au monde pour soi et ceux qui nous entourent, alors les rescapés sont ceux qui ont réussi à conserver au mieux, de manière follement restreinte, cette double donation de sens, celle qui permet à l'être humain de mettre le monde à sa mesure physiquement et intellectuellement et de contrôler autant que faire se peut toute situation et celle qui pourrait advenir (Hoyaux, 2002 ; 2003).

En cela, à la suite d'Hannah Arendt, «il conviendra de poser cette question importante : dans quelle mesure des êtres humains vivant sous la terreur totalitaire répondentils encore à la représentation que nous nous faisons habituellement de l'homme ? » (Arendt, 1990, 177).

\footnotetext{
${ }^{1}$ Je me permets de dédier cette note d'intention à Mme Suzanne Mathieu épouse Guimbretière et à sa mère, Justes devant les nations, pour service rendu à l'humanité aimante durant la Seconde Guerre mondiale.
} 
La question ici posée n'a pas bien entendu pour objectif de revenir sur les débats houleux entre historiens sur la question de la spécificité historique de l'extermination des Juifs, des Tsiganes ou des déficients mentaux et physiques allemands à partir de 1933 et jusqu'à la fin de la Seconde Guerre mondiale. En effet, le camp de concentration (Konzentrationslager) se distingue du camp d'extermination par le fait même qu'on n'y détruit pas systématiquement la personne physique. Au sens de Merleau-Ponty, on peut dire qu'on tente de détruire sa chair ou sa corporéité mais pas forcément son corps physique (Dastur, 2001). Ainsi, dans le camp de concentration, si l'on ne détruit pas l'homme on tente de détruire son humanité, c'est-à-dire les traits de sa personnalité, de sa volonté, de sa singularité.

Et cette humanité, c'est le fondement ontologique de l'habiter, au sens certes restreint d'un contexte épistémologique qui relève des analyses existentialistes. Elles sont donc soustendues par les concepts de monde et d'être-au-monde. Selon ces derniers, chaque être humain a toujours déjà un monde et se relie à lui dès sa naissance et en tout instant de son existence. Cependant, ce monde n'est pas compris comme un monde clos, une cage ontologique pour reprendre les termes de Peter Sloterdijk, mais un monde ouvert que l'être construit par ses pratiques et ses pensées, réelles ou imaginées.

Si l'habiter s'origine dans l'idée d'être-au-monde, c'est-à-dire d'être projeté au devant de lui-même en se tenant debout (ek-sistere) face au monde, en le construisant et en se réalisant en son sein par-delà l'horizon perceptible ; il devient pertinent de montrer comment le système totalitaire nazi va produire que ce soit pour les déportés ou les non déportés un monde clos sans perspective autre que celle qu'on leur impose.

Certes, ce sont les camps de concentration qui représentent les points extrêmes de cette systématisation d'enfermement physique et mental mais le monde "normal » sera lui aussi sous le joug de cette systématisation (Fallada, [1965] 2002 ; Klemperer, [1995] 2000 ; [19951996] 2000). Car celle-ci a pour objectif ultime de biologiser le monde, de le rendre métaphoriquement bestial (Mosse, [1990] 1999) pour le rétrograder au stade d'environnement (au sens existentialiste) et dénier à tous les êtres (déportés ou non) d'avoir leur monde, donc une humanité qui va au-delà des forces de l'habitude mécaniste, déterministe et centripète.

Cette attitude volontariste de ségrégation et de cloisonnement pratique et pragmatique qu'imprime par essence le régime totalitaire a ainsi pour finalité de naturaliser les conditions des différents existants, notamment selon les races pour le nazisme (Traverso, $2001 ; 2002$ ). Cette naturalisation est supposée conduire les uns à vivre uniquement sous le déterminisme des processus biologiques de l'animalité (dans les camps, les ghettos), et les autres sous le déterminisme des procédures intellectuelles soumises à la «tyrannie de la logique» de l'homme supposé supérieur (Arendt, [1951] 1972, 223).

Pour éclairer ce double fondement de l'habiter, l'analyse devra montrer comment le régime nazi a systématisé dans les camps de concentration d'abord en Allemagne (Ulm, Dachau, Nohra, Lugau, Plaue, Orianenburg), puis en Pologne (qui seront souvent associé à des camps d'extermination dès 1941) et dans l'ensemble des pays sous l'influence du III ${ }^{\text {ème }}$ Reich, des techniques physiques et psychiques pour empêcher toute possibilité d'habiter le monde pour les déportés (d'abord des prisonniers politiques puis essentiellement des Juifs ou des Tsiganes). Cette systématisation avait pour but d'ôter toute possibilité à ces derniers d'exister au sens phénoménologique du terme (et non pas forcément au sens biologique du vivre), c'est-à-dire d'empêcher qu'il puisse être acteur au moins pour partie de leur réalité, celle du monde dont chaque être s'entoure —, et de se réaliser en tant qu'être dans celui-ci. 


\section{La violence nazie : généalogie et/ou ontologie européenne?}

La question des camps nous amène aussi à repenser le sens de la violence inhérente au régime nazi. Doit-on positionner cette violence dans une généalogie (Traverso, 2002), dans un contexte historique précis ou doit-on au contraire y voir les signes de manières d'être et de faire plus profondément ancrées dans les habitus culturels d'une nation européenne (Sofsky, [1996] 1998), dans une ontologie ? Car au regard des fondements idéologiques de cette nation, - si tant est qu'elle existe en tant que tel -, on assiste à une quête effrénée de la totalité, territoriale et géopolitique, philosophique et morale, religieuse, etc. C'est dans l'orbe de cette tyrannie de la logique, d'une argumentation qui se satisfait à elle-même, que se sont toujours énoncées les réalités des camps de concentration. Hannah Arendt rappelle qu'ils « existaient avant que le totalitarisme n'en fasse l'institution essentielle de cette forme de gouvernement. Ils sont apparus, pour la première fois, pendant la guerre des Boers, et la notion juridique d'"internement de protection" a d'abord été utilisée dans les pays gouvernés de manière impérialiste, l'Inde et l'Afrique du Sud. Ce terme a servi par la suite, en raison de son caractère équivoque, de fondement "juridique" à toutes les arrestations effectuées en Allemagne nazie pour internement dans les camps de concentration : on pouvait l'interpréter comme signifiant la protection de la société vis-à-vis de la population des camps ou bien la protection de cette dernière contre la prétendue "colère du peuple" » (Arendt, [1972-1982] 1990, 175).

Les camps sont donc présentés initialement comme des zones de rétention administrative, de «rééducation » pour reprendre les termes d'Himmler dès mars 1933, des zones combinées de protection pour ceux qui sont dans et hors des camps. On protège seulement les uns des autres en leur refusant - d'un prétendu commun accord tacite - toute relation d'altérité, avec un monde autre, une pensée autre, un alter, relation qui préfigure justement le principe même de l'habiter. La limite étanche qui fonde la systématisation du rejet de l'altérité se trouve alors inscrite par l'idéologie dominante comme fonction secondaire de l'identité. Celle-ci, fondée sur la race, la classe, la nation détermine ce qui est à rejeter, à refuser, à impenser!

De ce fait, même si dans une certaine conception culturaliste, on peut soulever l'objection que comparaison n'est pas raison, et s'il faut bien entendu se garder de tout moralisme et de tout poncif bien-pensant, n'est-il pas possible d'émettre l'idée que nombre de femmes et d'hommes sont encore aujourd'hui mis à l'écart pour le bien-être des autres, pour éviter leurs colères (celles de payer trop d'impôts, de payer pour ces autres, mais surtout d'en avoir peur) et leur représailles : Immigrants, personnes âgées, déficients mentaux, handicapés physiques, SDF, prisonniers, chômeurs, etc. Cette possible comparaison, d'essence ontologique, tient des fondements mêmes de la société qui, comme le rappelle avec acuité Wolfgang Sofsky, «n'est fondée ni sur un besoin irrésistible de sociabilité ni sur les nécessités du travail [mais bien plutôt sur] l'expérience de la violence qui réunit les hommes. La société est un dispositif de protection mutuelle. Elle met fin à l'état d'absolue liberté. Désormais, tout n'est plus permis. [...] La guerre de tous contre tous ne consiste pas en un bain de sang perpétuel, mais à en avoir peur sans arrêt. Ce qui déclenche et fonde la socialisation, c'est la peur qu'ont les hommes les uns des autres » (Sofsky, [1996] 1998, 12). Dès lors, «le constant accroissement de la violence tient bien plutôt au projet même de l'ordre. La campagne contre toute déviance, l'expulsion des marginaux, la persécution des étrangers, tout cela était inscrit d'avance dans la charte fondatrice du pouvoir » (Sofsky, [1996] 1998, 17). 
Cette mise à l'écart relève de la lente et inéluctable re-construction d'un Monde total (Freitag in Dagenais 2003 ; Pinard in Dagenais, 2003) voué à l'assentiment de la sphère économique. Et cela au sens où les femmes et les hommes mis à l'écart de notre société mondialisée sont observés, expliqués, compris comme des flux d'êtres déséconomiquement externes. Leur existence est abolie par les membres d'un système absurde, sourd à leur nonconformité prétendue. Non-conformité, qui pour l'être conforme, appelle à une destruction administrative: ces derniers ne devant pas obtenir de carte de sécurité sociale, de carte d'identité, de permis de conduire, etc. payé par la collectivité. Il coûte trop cher au système monde post-historique, post-critique, post-réflexif, total ! Il ne rentre pas dans les cases que la société informatisée, nanotechnologique, génétique s'évertue de concocter pour notre supposé bien-être: gain de temps, gain de poids, gain de sécurité, gain d'argent surtout. Mais pour qui fondamentalement? Pour quel être ?

Car ce monde total, normal, engramme en chaque individu l'esprit de la dé-mesure, et renvoie son existence à l'incommensurable d'une seule idée, celle de la conformation de ses problèmes et de ses solutions, de ses espoirs et de ses désirs à la rationalité instrumentale. Et c'est cette rationalité instrumentale, cette machinerie institutionnelle qui doit nous inviter à réfléchir aujourd'hui à ces nouvelles formes de violence que connait l'habiter européen, celle du tout sécuritaire (Le Monde Diplomatique, 2003), et celle de la lente fascisation des esprits vouée à des néo-nationalismes identitaires (Duplan, 2003 ; Tévanian et Tissot, [1998] 2002) qui rappelle parfois quelques figures sociologiques de l'avant seconde guerre mondiale (Burrin, [1986] 2003). La première forme de violence recourt à l'idée d'ordre, la seconde, qui lui est sous-jacente, à celle de la norme.

D'un côté, « le rêve de l'ordre, c'est le rêve d'éliminer totalement l'ambivalence, le rêve d'une parfaite transparence, d'une société en verre. Rien ne doit échapper à l'œil des gardiens, car même le plus petit événement pourrait être la cellule germinale d'une subversion » (Sofsky, [1996] 1998, 21). Cet œil des gardiens semble s'imposer à chaque individu mais en fait il se l'impose le plus souvent à lui-même, par le truchement, l'excuse de la décision collective, car il ne sait pas se responsabiliser, se poser à lui-même ses propres limites imaginaires, abstraites. C'est en ce sens que l'homme crée des techniques de surveillances de plus en plus performantes : caméras urbaines, radars automatiques, bureaux vitrés des entreprises, cartes d'identité avec photos et bientôt empreintes digitales et codes génétiques, bracelets électroniques des prisonniers. Ces techniques de surveillance s'innervent dans le voyeurisme actuel, ce panoptisme à bon marché, qui permet à chacun d'entre-nous de contrôler l'autre et de nourrir en boucle rétroactive son impression d'accéder à des formes de pouvoir sur celui-ci. Cette immatérialisation des techniques de surveillance remplace la matérialisation ancestrale de la limite, celui du front pionnier comme celui des barbelés, celui des Etats comme celui des camps de concentration (Razac, 2000). Car cette frontière physique et symbolique que les institutions de contrôle ont toujours voulu inscrire dans l'espace pour s'approprier et défendre leur territoire avait justement pour but de créer le dans et le hors, le connu et l'inconnu, le sécurisé et le dangereux dans l'esprit des communautés humaines... se sentant homogènes.

De l'autre côté, "l'ordre, cela signifie la définition de normes et de la normalité, la production d'une uniformité, l'exclusion et l'oppression de quelque différence que ce soit » (Sofsky, [1996] 1998, 17). Il faut générer de l'homogénéisation dans les façons d'être et de vivre, et dans les significations que les hommes donnent à ces façons d'être et de vivre, c'est la culture du cercle herméneutique qui s'anoblit de lui-même puisque l'individu ne comprend jamais une réalité qu'avec les cadres interprétatifs dont il dispose. Ainsi, «avant la norme, rien ne détermine ce qui est normal ou anormal. La mesure prise produit elle-même les 
occasions qu'elle a de s'appliquer » (Sofsky, [1996] 1998, 21). Promulguer des lois, c'est alors imprimer la mesure étalon de nos interprétations dans «le gigantesque mécanisme de régulation » qu'instille le pouvoir politique qu'il soit démocratique ou totalitaire. C'est créer la décharge qui va orienter les significations que les membres d'un groupe «élu » donnent à leur relation au monde. "C'est l'instant où tous ceux qui en font partie se défont de leurs différences et se sentent égaux » (Canetti, [1960] 1966, 14). Si la mécanique nazie fonctionne aussi bien dès les années 1930, c'est justement parce que chaque discours d'Hitler est une décharge qui conforte et conforme les décisions du peuple allemand.

Mais au-delà, le simplisme herméneutique, qui ne se fonde d'ailleurs pas forcément sur le terreau de l'ignorance, mais bien plutôt sur la lassitude collective face à la l'impossible compréhension de la totalité complexe des mondes sociaux, économiques et politiques d'une période donnée, aboutit immanquablement à la radicalité des langages de ceux qui tiennent le pouvoir. Et malheureusement, " aucun langage n'est plus convaincant que le langage de la violence. Il n'a pas besoin de traduction et ne laisse aucune question en suspens » (Sofsky, [1996] 1998, 20).

Les débats historiographiques qui se nourrissent sur la question de savoir si le nazisme était un fascisme ou un totalitarisme paraissent donc superfétatoires à l'aune de la pensée sur l'habiter et de son évolution préoccupante. Sans aller jusqu'à déclarer que l'habiter contemporain relève des mêmes symptômes et déviances de l'habiter tel que mis en place dans les camps de concentration, on peut toutefois se demander si la généalogie de la violence nazie tel que l'énonce Enzo Traverso (2002) ne préfigure pas les différents types de violence qu'elle soit physique ou symbolique que nous connaissons aujourd'hui et qu'avait déjà analysé avec pertinence Sofsky ([1996] 1998 ; 2002), Bourdieu $(1994 ; 2001)$ ou Foucault (1975).

Sans vouloir aucunement choquer la mémoire des personnes ayant connu la shoah, il est temps de se demander en quoi le camp de concentration n'est pas l'un des signes précurseurs du fondement ontologique de notre société actuelle, celui d'une mise en place instrumentale - autant intellectuelle que technique - d'une "violence civilisée", celle d'un "fétichisme de la science » et de la réalisation de "projets de planification autoritaire et d'ingénierie sociale » (Traverso, 2001, 19). Cette planification économiciste s'ancre dans les valeurs bureaucratiques des pays développés dont Joseph Billig [1967] 2000 et Zygmunt Bauman (2002) notent qu'elles sont au cœur de la société allemande des années 1930 par l'intermédiaire de son système industriel. Ce système industriel englobe le système concentrationnaire, philosophiquement, - car il recourt au même volonté d'ordre, de hiérarchie - et matériellement car c'est l'homme qui en devient la matière première. Pour ces auteurs, la shoah n'est donc pas un accident de l'histoire, une tragédie interne à l'histoire juive, mais bien plutôt le signe tangible d'un usinage de nos mentalités. Car ces projets de planification sont systématiquement associés à une batterie argumentative sur leurs bienfaits supposés pour l'habiter des populations élues ou non élues, ainsi le travail ne rendait-il pas libre (Arbeit macht frei pouvait-on lire à l'entrée des camps). L'habiter est alors compris au sens le plus caricatural d'une meilleure qualité de vie, expurgée des scories sociétales que seraient les figures génériques de «l'étranger», du «pauvre », du «vieux », du « fou », du «libertaire », devenues catégories de l'anormalité dans la culture européenne... (Foucault, 1999).

Dans cette batterie argumentative, d'anciennes terminologies - instinct, peuple, mondial, vision du monde (weltanschauung), étranger à l'espèce -, semble ainsi s'associer à de plus récentes - naturellement, terroir, système, réseau, insécurité, immigration - pour 
perpétuer implicitement, voire de plus en plus explicitement, des langages totalitaires (Faye, 1972 ; Klemperer, [1947] 1996) qui ont pour objet de normer les réflexions de tous les citoyens sur ce que serait justement ce bon habiter et le bon habitant qui va avec. Mais l'argumentation n'est pas une composante récente de notre humanité. La rhétorique grecque inscrivait déjà cet espace de la discrimination idéologique. Pour autant, elle est ici inscrite comme une des figures tutélaires majeures de la violence car elle s'incarne, de même que la mise en scène architecturale (Abensour, 1997), comme le média par excellence du pouvoir qui en est justement l'implicite de sa raison d'être (Sofsky, [1996] 1998).

L'étude sur l'habiter dans les camps de concentration sous le nazisme englobe donc de nombreuses facettes - philosophique, géographique, politique, idéologique, psychologique, sociologique, historique - qui interagissent entre-elles pour construire la situation abordée. Et nous sommes conscients que le champ proposé ici est très clairement restrictif tant par sa dimension épistémologique que par son contexte spatial (camp de concentration) et temporel (sous le régime nazisme), - mais peut-il en être autrement tant le champ d'investigation des totalitarismes et des entreprises génocidaires dans l'histoire est vaste et délicat - il permet cependant de développer les fondements même de l'habiter, et au-delà des figures de la violence dans la construction des territoires (Sofsky, 2002).

Pour aborder ce champ d'analyse, la démarche méthodologique doit s'inscrire autant sur des recherches d'historiens et spécialistes de la question concentrationnaire (Bensoussan, 1998 ; Billig, [1967] 2000 ; Brossat, 1996 ; Sofsky, [1993] 1995 ; Traverso, 2002 ; Wieviorka, 1992 ; 2005) que sur d'éminentes descriptions et interprétations des produites par certains de ceux qui ont réussi à réchapper d'Auschwitz, de Dachau ou de Buchenwald pour les camps les plus connus tels que Bruno Bettelheim ([1960] 1972), Primo Lévi ([1958] 1987 ; [1995] 2000), David Rousset ([1946] 1965), Anne-Lise Stern (1994) ou Stanislav Zámecník ([2002] 2003), mais aussi de camps plus «insignifiants » à nos mémoires communes (Gandersheim) mais dans lesquels pour autant l'horreur y était aussi «obscurité, manque absolu de repère, solitude, oppression incessante, anéantissement lent » (Antelme, 1957). Enfin, il est à noter que d'autres formes de récits, notamment documentaires (Shoah de Claude Lanzmann; De Nuremberg à Nuremberg de Frédéric Rossif; La libération des camps nazis, etc.), voire filmiques (Nuit et Brouillard d'Alain Resnais, La liste de Schindler de Steven Spielberg, Amen de Costa Gavras, Le Pianiste de Roman Polanski), rendent utilement service à la compréhension qualitative (car relative à la mémoire que ceux qui restent en ont) des faits et des actes, des sentiments et des affects, même s'ils sont parfois teintés de moralité et d'idéologie. A côté de la rudesse des chiffres et des récits, ils animent cette abstraction que peut parfois représenter l'horreur et l'absurdité et dans laquelle les « Juifs », les « Tziganes », les « Noirs » étaient confinés.

\section{Briser les constructions territoriales et la constitution ontologique de l'homme}

Comme nous l'avons dit, la construction territoriale relève de cette nécessité pour l'homme de se relier à des entités spatialisées, socialisées et temporalisées qui sont visibles dans sa réalité présente ou dans sa réalité mnémonique. Cette relation permet à l'homme de construire son identité d'être humain, d'en délimiter son contour corporel, de son corps à son champ d'action. Elle permet ainsi de créer en lui des niveaux d'investissement du monde spatial, social et temporel selon différents champs et seuils qui fondent ses "logiques topologiques". Ces dernières « entourent nos définitions de ce qui est à moi/à toi, privé/public, autochtone/étranger, proche/lointain, central/périphérique...: toutes ces représentations personnelles, qui changent sociétalement, et qui ont tant d'importance dans nos relations interpersonnelles. Là se trouvent les fondements de tant de nos lois et règlements, la source 
des codes de nos pratiques qui savent être conviviales, comme de nos recours à l'arbitrage ou à la force; le fondement aussi des conditions nécessaires et/ou suffisantes pour que soit garantie effectivement l'autonomie de chaque "agent" concerné » (Ferrier J.-P., 1986, 5).

La construction territoriale permet donc à l'homme de créer cette extensivité territoriale délimitée par ses soins qui lui assure une forme de liberté d'expression (champ des significations à toute fin pratique) et de mouvements (champ des pratiques à toute fin signifiante). Dans le cas des camps de concentration, l'analyse tentera de montrer que l'idée des concentrationnaires est justement de briser cette possibilité d'extensivité territoriale choisie. En effet, pour ces derniers, l'objectif est de briser tout possibilité pour l'être humain de se projeter sur un monde autre que celui du camp, c'est-à-dire d'empêcher la mise en place de toute relation physique ou psychique à quelque chose (des objets, des routines, des souvenirs) ou avec quelqu'un (les membres de sa famille, les autres déportés), et cela en concentrant systématiquement toutes les dimensions de ses territoires au seul camp de concentration, mais aussi en uniformisant ses temporalités en une non-réalité du présent, inscrite comme l'instant inéluctable où l'on fait ce que l'on nous dit de faire sans poser de question, sans offrir alors la possibilité de donner du sens à ce que l'on fait et ce que l'on est.

Pour analyser cette systématisation totalitaire des camps, il est intéressant de présenter de manière systématique les différentes formes de violence physique et mentale qui avaient pour but de briser les constructions territoriales. Là aussi, l'analyse trouve sa positivité dans la mise en lumière des éléments qui ont permis à celles et ceux qui en ont réchappés, de comprendre en fait comment ils ont fait pour s'en sortir (dans tous les sens du terme), c'est-àdire pour se projeter hors de ce néant, de cette déréalisation territoriale du camp et poursuivre l'expressivité de leur être-au-monde dans ces anti-espaces et ces anti-temps.

Pour illustrer ce propos, il est possible de présenter de façon introductive deux exemples significatifs proposés par Bruno Bettelheim sur l'organisation scrupuleuse mise en place par les SS pour anéantir des deux principales dimensions de l'habiter : la construction territoriale et la constitution ontologique de l'être humain.

D'un côté, il montre que la construction du monde des déportés au fil du temps se trouve inscrite dans une spirale centripète qui se coupe progressivement de la réalité des anciens territoires pratiqués par ces derniers: "A la différence, caractéristique, des 'nouveaux', les 'anciens' n'étaient plus capables d'évaluer correctement le monde extérieur, non dominé par la Gestapo. Alors que les nouveaux prisonniers persistaient à considérer le monde du camp comme irréel pour les 'anciens', il était devenu la seule réalité. La conscience de la réalité du monde extérieur dépendait en grande partie de la force des liens affectifs du prisonnier avec sa famille et ses amis, de la vigueur et de la richesse de sa personnalité et de sa capacité à préserver des centres d'intérêt et des attitudes qui avaient eu de l'importance pour lui. Plus ses centres d'intérêt étaient variés, et plus il cherchait à en tirer parti dans le camp, plus il parvenait à protéger sa personnalité contre un appauvrissement trop rapide. [...] Lorsqu'on demandait aux "anciens" pourquoi ils parlaient si peu de leur avenir hors du camp, ils admettaient fréquemment qu'ils n'étaient plus capables de s'imaginer vivant dans un monde libre, prenant des décisions, s'occupant d'eux-mêmes et de leur famille " (Bettelheim, [1960] 1972, 219-220).

D'un autre côté, il montre que la constitution ontologique se trouve annihilée par la perte de la volonté de tout engagement ou de toute prise de responsabilité. Cette perte de volonté est le signe tangible d'une déstructuration de toute forme de conception idéologique (religieuse, politique, etc.) personnelle sur soi et le monde. Celle-ci passe d'abord par la perte de la liberté, mais par l'absurdité des actions engagées par les SS et puis par les déportés eux- 
mêmes sous la contrainte du camp. Ainsi, toute possibilité de faire sens pour soi est niée par les SS, tant au niveau du sens de l'action des SS par rapport aux déportés qu'au niveau du sens de l'action de l'être par rapport à lui-même. Cette interdiction s'inscrivait même sur l'acte ultime, celui d'organiser sa propre mort. A priori, pour les SS, dont le rôle était à terme d'exterminer les Juifs ou les Tsiganes; "plus le nombre des suicides était élevé, plus leur besogne en était facilitée. Mais, même dans ce cas, la décision ne devait pas venir du prisonnier. Un S.S. pouvait provoquer un prisonnier à se tuer en se jetant contre le grillage électrifié de la clôture. Mais si un prisonnier tentait de se tuer de sa propre initiative et n'y réussissait pas, le règlement, édicté à Dachau en 1933, prévoyait qu'il recevrait vingt-cinq coups de fouet et serait mis au cachot pendant une période prolongée. On peut penser qu'on punissait ainsi son échec. Mais [...] il s'agissait beaucoup plus de punir un acte d'autodétermination." (Bettelheim, [1960] 1972, 205-206).

Ainsi, à côté de la violence physique, les concentrationnaires utilisaient les dispositifs les plus extrêmes de la violence symbolique, c'est-à-dire la possibilité offerte à tout régime totalitaire de mettre en conformité une population entière vis-à-vis des "us et coutumes"... "aléatoires"! de quelques-uns, en l'occurrence les gardiens SS des camps.

L'analyse tentera donc de montrer qu'au-delà de la cruauté des tueries, de l'absurdité réfléchie de l'appareil tortionnaire conditionnant le hasard de la survie des uns plutôt que des autres (Lévi P., [1995] 2000), les seuls êtres humains qui ont réussi à résister aux camps de concentration ont été ceux qui ont su préserver autant que faire se peut une part de leur habitation, c'est-à-dire une part de leur relation avec un monde, le leur et non seulement celui du camp et une part de relation avec eux-mêmes et non seulement avec leur tortionnaire et le non-sens de leur action.

La discrimination permet à l'homme de structurer sa sécurité ontologique autour des valeurs idéologiques qu'il se donne. Lui ôter cette possibilité, c'est lui ôter la possibilité de stabiliser toute réflexion sur la construction de la réalité qu'il a configuré avec le monde spatial, social et temporel qui l'entoure. C'est donc lui ôter toute possibilité de se projeter dans son monde. L'idéologie de la terreur a pour principe au-delà de la violence physique d'annihiler toute possibilité de construction mentale de la réalité.

\section{L'inhabitabilité du nazisme}

Le régime nazi, comme tous les régimes totalitaires, a créé l'annihilation des besoins soit en les contentant (pour le peuple) soit en les supprimant (pour les déportés). Et c'est en cela qu'il a également détruit le temps et l'espace des hommes pour le remplacer par ceux du mythe, de l'identité factice de la race élue en ôtant toute pensée de l'autre.

Car l'être humain en habitant désire se construire un monde dont il dépend. Et ce sont les dimensions mêmes de cette dépendance qui délimitent le champs de son habitation et de son inhabitation du monde. S'il est préoccupé par quelque chose, dans l'attente de quelqu'un, dans l'extase permanente vers un monde, il est aussi pétri d'habitudes, de certitudes qui sont autant de barrières à cette sortie dans le hors-là. Cette force de l'habitude détermine des façons d'être, de faire et de penser qui se sédimentent plus ou moins au cours des événements, qui au gré des complexes possibles s'affranchissent ou non des situations et de ce qu'on leur fait dire de manière normative, unipolaire et hermétique. Pourtant, "le danger d'échanger la nécessaire insécurité où se tient la pensée philosophique pour l'explication totale que propose une idéologie et sa Weltanschauung, n'est pas tant le risque de se laisser prendre à quelque postulat généralement vulgaire et toujours pré-critique, c'est celui d'échanger la liberté inhérente à la faculté humaine de penser pour la camisole de la logique, avec laquelle 
l'homme peut se contraindre lui-même presque aussi violemment qu'il est contraint par une force extérieure à lui" (Arendt, [1951] 1972, 218).

Penser l'habiter, c'est penser l'idéologie morale et philosophique qu'elle génère. Ainsi, pour Heidegger, habiter c'est "être mis en sûreté [...] rester enclos dans ce qui nous est parent, c'est-à-dire dans ce qui est libre et qui ménage toute chose dans son être. Le trait fondamental de l'habitation est ce ménagement" (Heidegger, [1954] 1958, 176). Derrière ce ménagement, il y a l'idée d'une acceptation tautologique pour l'être, d'être ce qu'il $a$ à être au sein de l'être de ce qu'il a. Mais encore faut-il concevoir la finalité - si finalité il y a - de l'avoir à être et la déterminité - l'authenticité dirait Heidegger - de l'être de ce qu'il a. Ce sont cette finalité et cette déterminité qui posent problèmes car leurs interprétations diffèrent d'un être, d'un collectif à l'autre, d'un vécu contingent à l'autre. Sur celles-ci, chacune et chacun peut développer ses idées, et celles-ci sont le plus souvent pensées dans et comme une totalité. Elles nourrissent un champ d'interprétations, des réalités qui nous entourent, qui se trouve toujours déjà confiné en lui-même dans un cercle herméneutique. Le problème de ce confinement idéologique, c'est que s'il apporte la nécessaire sécurité dont les êtres humains ont besoin pour affronter le Monde et construire leur monde en son sein; il généralise les phénomènes et les événements qui se sont déroulés sous la logique d'une idée avant d'en totaliser et d'en naturaliser l'efficience générative pour tous les événements et phénomènes à venir.

De ce point de vue, l'erreur heideggérienne tient à cette fonction hermétique, par principe cloisonnante et normative, qu'il a attaché au sens de l'être, au revers de ce concept d'authenticité et d'obéissance au destin. On y retrouve la force ambivalente des mots noués dans la Kriegsideologie (Communauté, mort, destin) et transformée en « puissance » des idées par le régime hitlérien qui s'est repaît jusqu'à plus soif de cette compromission parfois naïve des intellectuels allemands (Losurdo, 1998).

Car comme le rappelle Arendt, en réalité, «les idéologies ne s'intéressent jamais au miracle de l'être. Elles sont historiques, occupées du devenir et du disparaître, de l'ascension et de la chute des cultures, même si elles essaient d'expliquer l'histoire par quelque "loi naturelle". Le mot 'race' dans racisme ne signifie aucunement une curiosité authentique au sujet des races humaines en tant que domaines d'exploration scientifique; il est une idée qui permet d'expliquer le mouvement de l'histoire comme un processus unique et cohérent » (Arendt, [1951] 1972, 216-217). Dans le cas des camps de concentration, l'acceptation commune de cette idéologie - celle des gardiens mais aussi à la longue celle des prisonniers accélère la naturalisation de cette réalité imaginée, mythifiée et génère la perte définitive de tout ancrage territorial pour les déportés.

Ainsi, ce sont vraisemblablement les femmes et les hommes qui ont su se départir de leur sécurité ontologique, c'est-à-dire de leur cercle herméneutique initial, et modifier ainsi leur champ d'interprétation des événements non plus à l'aune de la déterminité et de la finalité de l'essence philosophique, religieuse, morale de leur présence sur Terre, tel qu'il la concevait jusqu'alors, qui ont réussi à survivre dans les camps de concentration. Ils ont réussi à garder un monde auquel se relier, auprès duquel espérer. Ce monde construit passait parfois par un égocentrisme sécuritaire et il est d'ailleurs à noter le sentiment de culpabilité qui s'exprime très souvent chez les rescapés. Sentiment non pas seulement fondé sur le fait de cette incompréhension d'avoir eu de la chance par rapport à d'autres membres de leur famille, de leur clan ; mais aussi d'avoir été autrement que ce qu'il/elle pensait être, de s'être vu faire ou dire des choses qu'il/elle ne pensait pas faire ou dire (Bettelheim, Levi, Stern). Être différent de l'humain que l'on croyait être en tentant pour autant de ne pas être étranger à soi-même 
pendant et après l'abomination. C'est dans cet interstice, au-delà du raisonnable, de l'explicable a posteriori que naît la souffrance de ce qui ont vécu les camps. C'est dans cette ab-surdité que l'être ne veut parfois plus s'entendre et se rendre peut-être, comme Primo Levi, sourd à lui-même (Cravetto, 2000).

\section{Bibliographie Introductive}

Tonka.

Abensour M., 1997, De la compacité. Architectures et régimes totalitaires, Paris, Sens \&

Antelme R., 1957, L'espèce humaine, Paris, Gallimard.

Arendt H., [1951] 1972, Les origines du Totalitarisme : 3. Le système totalitaire, Paris, Le Seuil.

Arendt H., [1963] 2002, Eichmann à Jérusalem. Rapport sur la banalité du mal, Paris, Éditions Gallimard.

Arendt H., [1972-1982] 1990, La nature du totalitarisme, Paris, Éditions Payot.

Audi P., 2004, Où je suis : Topique du corps et de l'esprit, La Versanne, Encre Marine.

Bauman Z., [1999] 2002, Le coût humain de la mondialisation, Paris, Hachette Littératures.

Bauman Z., 2002, Modernité et Holocauste, Paris, La Fabrique Éditions.

Bensoussan G., 1998, Auschwitz en héritage? D’un bon usage de la mémoire, Paris, Éditions Mille et une nuits. Littératures.

Bettelheim B., [1960] 1972, Le Cour conscient, Paris, Éditions Robert Laffont - Hachette Plumes.

Bilé S., 2005, Noirs dans les camps nazis, Monaco, Les Éditions du Rocher / Le Serpent à

Billig J., [1967] 2000, L'Hitlérisme et le système concentrationnaire, Paris, PUF.

Bourdieu P.1994, Raisons pratiques. Sur la théorie de l'action, Paris, Éditions du Seuil.

Bourdieu P., 2001, Langage et pouvoir symbolique, Paris, Éditions du Seuil. Michel.

Brossat A., 1996, L'épreuve du désastre. Le XXème siècle et les camps, Paris, Éditions Albin du Seuil.

Burrin P., [1986] 2003, La dérive fasciste. Doriot, Déat, Bergery. 1933-1945, Paris, Éditions

Canetti E., [1960] 1966, Masse et puissance, Paris, Éditions Gallimard.

Chesnaux J., 1996, Habiter le temps, Paris, Bayard Éditions.

Cravetto M.L., 2000, Fidélité à l'après. A propos du suicide de Primo Levi et de l'intériorité du Mal, Paris, Éditions Kimé.

Dagenais D. (dir.), 2003, Hannah Arendt, le totalitarisme et le monde contemporain, Québec (Canada), Les Presses Universitaires de Laval.

Dastur F., 2001, Chair et langage : Essais sur Merleau-Ponty, La Versanne, Encre Marine.

Dorra M., 2001, Heidegger, Primo Levi et le séquoia. La double inconscience, Paris, Éditions Gallimard.

Ducher G., 2005, Les camps tragiques, Paris, Éditions Cartouche.

Duplan C., 2003, Mon village à l'heure Le Pen, Paris, Éditions du Seuil.

Fallada H., [1965] 2002, Seul dans Berlin, Paris, Éditions Denoël.

Faye J.-P., 1972, Langages totalitaires, Paris, Hermann.

Ferrier J.-P., 1986, «Habiter/Penser la Terre: géographie et idée de l'avenir », Méditerranée : Revue géographique des Pays Méditerranéens, ${ }^{\circ}$ 3, tome 58, p. 3-10.

Filhol E., [2001] 2004, La mémoire et l'oubli : L'internement des Tsiganes en France, 19401946, Paris, L'Harmattan / Centre de recherches tsiganes.

Foucault M., 1975, Surveiller et punir. Naissance de la prison. Paris, Éditions Gallimard.

Foucault M., 1999, Les anormaux. Cours au Collège de France 1974-1975, Paris, Gallimard /

Seuil.

Graumann C. F., 1989, "Vers une phénoménologie de l'être-chez-soi", Architecture \& Comportement, 5 - 2, p. 111-116.

Heidegger M., [1927] 1964, L'Etre et le Temps, Paris, Gallimard. 
Heidegger M., [1954] 1958, Essais et conférences, Paris, Gallimard.

Hoyaux A.-F., 2002, "Entre construction territoriale et constitution ontologique de l'habitant. Introduction épistémologique aux apports de la phénoménologie au concept d'habiter ", Cybergéo: Revue européenne de géographie, $\mathrm{n}^{\circ} 216,18 \mathrm{p}$.

Hoyaux A.-F., 2003, "Les constructions des mondes de l'habitant : Éclairage pragmatique et herméneutique", Cybergéo : Revue européenne de géographie, $\mathrm{n}^{\circ} 232,23 \mathrm{p}$.

Hoyaux A.-F., 2003, "De l'espace domestique au monde domestiqué. Point de vue phénoménologique sur l'habitation", in Collignon B. et Staszak J.-F. (dir.), Espaces domestiques. Construire, habiter, représenter, Paris, Bréal Éditions, p. 33-45.

Keneally T., [1982] 1984, La liste de Schindler, Paris, Éditions Robert Laffont.

Klemperer V., [1947] 1996, LTI (Lingua Tertii Imperii). La langue du Troisième Reich. Carnets d'un philologue, paris, Éditions Albin Michel.

Klemperer V., [1995] 2000, Mes soldats de papier. Journal 1933-1941, Paris, Éditions du Seuil.

Klemperer V., [1995-1996] 2000, Je veux témoigner jusqu'au bout. Journal 1942-1945, Paris, Editions du Seuil.

Lacoue-Labarthe P. et Nancy J.-L., [1991] 1998, Le mythe nazi, La Tour d'Aigues, Éditions de l'aube.

Le Monde Diplomatique, 2003, « Obsessions sécuritaires », Manière de voir, $\mathrm{n}^{\circ} 71$.

Levi P., [1958] 1987, Si c'est un homme, Paris, Julliard.

Levi P., [1986] 1989, Les naufragés et les rescapés. Quarante ans après Auschwitz, Paris, Éditions Gallimard.

Levi P., [1995] 2000, Le Devoir de mémoire, Paris, Éditions Mille et une nuits, Entretien avec Bravo A. et Cereja F.

Losurdo D., 1998, Heidegger et l'idéologie de la guerre, Paris, PUF.

Mosse G.L., [1990] 1999, De la Grande Guerre au totalitarisme: La brutalisation des sociétés européennes, Paris, Hachette Littératures.

Ravaisson F., [1838] 1997, De l'Habitude, Paris, Éditions Payot \& Rivages.

Razac O., 2000, Histoire politique du barbelé, Paris, La Fabrique Éditions.

Rousset D., [1946] 1965, L'Univers concentrationnaire, Paris, Les Éditions de Minuit / Hachette Littératures.

Roux M., 2002, Inventer un nouvel art d'habiter. Le ré-enchantement de l'espace, Paris, L'Harmattan.

Serfaty-Garzon P., 2003, Chez soi. Les territoires de l'intimité, Paris, Armand Colin / SEJER.

Sloterdijk P., 2000, La domestication de l'être, Paris, Mille et une nuits (Arthème Fayard). Calmann-Lévy.

Sofsky W., [1993] 1995, L'organisation de la terreur. Les camps de concentration, Paris,

Sofsky W., [1996] 1998, Traité de la violence, Paris, Gallimard.

Sofsky W., 2002, L'ère de l'épouvante. Folie meurtrière, terreur, guerre, Paris, Gallimard.

Stern A.-L., 2004, Le savoir-déporté. Camps, histoire, psychanalyse, Paris, Éditions du Seuil.

Surya M. (dir.), 2000, « David Rousset », Lignes, Nouvelle série n 02, mai, Paris, Éditions Léon Scheer.

Taylor K., [1938] 1998, Inconnu à cette adresse, Paris, Éditions Autrement.

Tévanian P. et Tissot S., [1998] 2002, Dictionnaire de la lepénisation des esprits, Paris, L'esprit Frappeur.

Traverso E., [1995] 1997, Pour une critique de la barbarie. Ecrits sur l'histoire des Juifs et de l'antisémitisme, Lausanne, Éditions Page Deux.

Traverso E. (éd.), 2001, Le Totalitarisme. Le XXème siècle en débat, Paris, Éditions du Seuil.

Traverso E., 2002, La violence nazie, une généalogie européenne, Paris, La fabrique-éditions.

Van Camp H., 2003, Auschwitz oblige encore. Tentative pour penser le mal absolu à partir du bien toujours relatif, Paris, L'Harmattan.

Wieviorka A., 1992, Déportation et génocide. Entre la mémoire et l'oubli, Paris, Hachette.

Wieviorka A., 2005, Auschwitz, 60 ans après, Paris, Éditions Robert Laffont. 
Zámecník S., [2002] 2003, C'était ça, Dachau 1933-1945, Paris, Le Cherche-Midi / Fondation Internationale de Dachau. 